\title{
Relevance of Animal Models and Echocardiogram for Hypertensive Disease Studies
}

\author{
Karyne Pollo de Souza ${ }^{\circledR}$ and Christianne Brêtas Viera Scaramello \\ Universidade Federal Fluminense - Instituto Biomédico, Niterói, RJ - Brazil \\ Editorial related to the article: Electrocardiographic Abnormalities in Hypertension Models
}

Hypertension is the main risk factor for the development of cardiovascular diseases, such as cerebrovascular and ischemic heart diseases, as well as for premature death worldwide. The prevalence of this condition was about $30 \%$ in low-, middle- and high-income countries in 2010. Cardiovascular pharmacotherapy and lifestyle changes constitute important measures to manage cardiovascular diseases, and the reduction of blood pressure is a fundamental strategy to prevent and treat them. Systemic arterial hypertension is a clinical multifactorial condition. The underlying mechanisms of hypertensive disease are complex, which is why it is essential to elucidate them. ${ }^{1}$ Many animal models of hypertension can serve this purpose, most of which were developed considering the probable causes of this condition, such as high salt intake and renin-angiotensin-aldosterone system overactivity. It is important that each model exploit a single pathway to hypertension development. ${ }^{2}$

Spontaneously hypertensive rats (SHR) are genetically modified animals commonly chosen for several studies, including antihypertensive drugs screening. They provide an inexpensive experimental model and have a hypertensive phenotype similar to human patients. There are also endocrine models of hypertension, including the administration of deoxycorticosterone acetate (plus salt) and $\mathrm{N}-\omega$-nitro-L-arginine methyl- ester (L-NAME). According to the literature, both SHR and L-NAME models are related to sympathetic overactivity. ${ }^{2,3}$

A hypertensive condition can affect cardiac electrical activity and, since electrocardiogram (ECG) techniques

\section{Keywords}

Hypertension/complications; Blood Pressure; Cardiovascular Diseases/mortality; Electrocardiography/ methods; Rats; Animal Models. involve the recording of the heart's electrical activity, it seems to be a useful tool to evaluate high blood pressure-related cardiac impairment. These findings may explain the greater occurrence of arrhythmias and sudden death among hypertensive patients. ECG is considered a simple and inexpensive technique, being widely performed to diagnose cardiovascular diseases. ${ }^{4,5}$

The manuscript published by Conceição-Vertamatti et al., ${ }^{6}$ in the International Journal of Cardiovascular Sciences, proposes a comparative analysis encompassing two different experimental models of hypertension (endocrine/genetic) and their respective controls. Thus, the animals were divided into four experimental groups (6 animals/group): Wistar rats treated and untreated with L-NAME, Wistar-Kyoto rats and SHR. In brief, L-NAME treatment $(40 \mathrm{mg} / \mathrm{kg}$ / day in drinking water) was performed between postnatal weeks 10 and 15. All assays were conducted under anesthesia. The four experimental groups were submitted to ECG recording, both at postnatal weeks 6 and 15, while non-invasive blood pressure measurements were carried out only at the end of the experimental period. Data normality was confirmed (Kolmogorov-Smirnov test) followed by unpaired Student's t-test or the Mann-Whitney.

The authors observed higher systolic, diastolic, mean and mean diastolic pressures in L-NAME and SHR groups compared to their respective controls (Wistar and Wistar-Kyoto rats). In addition, SHR also presented higher time- to-peak values than Wystar-Kyoto rats. According to Conceição-Vertamatti et al., ${ }^{6}$ these data are in agreement with the literature and validate the experimental models of hypertension used. No differences were observed between controls or hypertensive groups regarding the hemodynamic parameters.

Several differences were noticed between SHR and Wistar-Kyoto rats regarding the ECG parameters,

Mailing Address: Christianne Brêtas Viera Scaramello

Rua Prof. Hernani Mello, 101. Postal Code: 24210-130, São Domingos, Niterói, Rio de Janeiro, RJ - Brazil.

E-mail: chrisbretas@gmail.com 
especially at postnatal week 6. This pattern was not observed when comparing Wistar with L-NAME rats, which presented few differences, only at postnatal week 15. The highest parameters found for the L-NAME group were corrected QT, QT and JT intervals, which suggests changes in ventricular repolarization. SHR presented higher RR/PR intervals, S/P/Q amplitudes and P duration, while corrected QT/QT/JT intervals, $\mathrm{R}$ amplitude and heart rate were decreased. ECG parameters were not distinguished between controls, although differences were noticed when comparing SHR and L-NAME groups. Because L-NAME treatment began at postnatal week 10, it is important to highlight the differences between them, observed only at postnatal week 15 (increased RR interval and $\mathrm{P}$ amplitude, decreased JT interval for SHR). These findings altogether suggest that SHR developed cardiac dysfunction, whereas L-NAME rats presented alterations in ventricular performance. Thus, depending on the experimental model, the adaptive mechanisms triggered by hypertension may be distinct. ${ }^{6}$

Previous researches have included these two hypertensive experimental models and the evaluation of ECG tracings. Scridon et al., ${ }^{7}$ reported unprovoked atrial tachyarrhythmias in aging spontaneously hypertensive rats correlating them with changes in the autonomic nervous system. Abdel-Rahman et al.,

\section{References}

1. Mills KT, Stefanescu A, He J. The global epidemiology of hypertension. Nat Rev Nephrol. 2020;16(4):223-37.

2. Leong X-F, Ng C-Y, Jaarin K. Animal Models in Cardiovascular Research: Hypertension and Atherosclerosis. Biomed Res Int. 2015; 528757.

3. Lerman LO, Kurtz TW, Touyz RM, Ellison DH, Chade AR, Crowley SD, et al. Animal Models of Hypertension: A Scientific Statement From the American Heart Association. Hypertension. 2019;73(6):e87-e120.

4. Reichlin T, Abächerli R, Twerenbold R, Kühne M, Schaer B, Müller C, et al. Advanced ECG in 2016: is there more than just a tracing? Swiss Med Wkly. 2016; 28;146:w14303.

5. Vilas-Boas F, Lima AAC, Torreão J, Feitosa GS. Dispersão Temporal do QT em Pacientes com Hipertensão Arterial Sistêmica. Arq Bras Cardiol. 1997; 68(5):343-6. in their turn, described differences between L-NAME and Wistar rats regarding the ECG parameters similarly to Conceição-Vertamatti et al., ${ }^{6}$ However, the latter authors highlighted the fact that none of them compared two different models of hypertension with each other on this matter.

Although of great importance, the manuscript published by Conceição-Vertamatti et al., ${ }^{6}$ presents a few limitations that must be briefly discussed. More accurate data could be obtained with a different strategy for oral administration of L-NAME, rather than the drinking-water approach. It is important to consider that the authors kept three rats in the same cage, so the gavage method should be preferred. In addition, since the authors performed multiple comparisons, one-way ANOVA and Kruskal-Wallis tests should also be preferred for the statistical analysis. Moreover, it would be interesting if the authors had included females in their study, since the literature describes that sex-related differences are observed in the progression of cardiovascular disease and survival. ${ }^{9}$

Studies are required to test strategies for hypertension prevention and control, especially among low-income populations. Therefore, studies that allow for a better understanding of the hypertensive pathology are very welcome.

6. Conceição-Vertamatti AG, Borghi F, Ishizu LY, Costa GT, Ramos LA, Areas MA, Grassi-Kassisse DM. Electrocardiographic Abnormalities in Hypertension Models. Int J Cardiovasc Sci. 2020; 33(4):321-328.

7. Scridon A, Gallet C, Arisha MM, Oréa V, Chapuis B, Li N, et al. Unprovoked atrial tachyarrhythmias in aging spontaneously hypertensive rats: the role of the autonomic nervous system. Am J Physiol Heart Circ Physiol. 2012;303(7):H386-H392.

8. Abdel-Rahman RF, Hessin AF, Abdelbaset M, Ogaly HA, AbdElsalam RM, Hassan SM. Antihypertensive Effects of Roselle-Olive Combination in L-NAME-Induced Hypertensive Rats. Oxid Med Cell Longev. 2017;2017:9460653. Doi:10.1161/JAHA. 119.014448. Epub 2019) Oct 30.

9. Siontis KC, Ommen SR, Geske JB. Sex, Survival, and Cardiomyopathy: Differences Between Men and Women With Hypertrophic Cardiomyopathy. J Am Heart Assoc. 2019;8:e014448 